\title{
ENUMERATING SUPER EDGE-MAGIC LABELINGS FOR THE UNION OF NONISOMORPHIC GRAPHS
}

\author{
A. AHMAD, S. C. LÓPEZ ${ }^{凶}$, F. A. MUNTANER-BATLE and M. RIUS-FONT
}

(Received 10 December 2010)

\begin{abstract}
A super edge-magic labeling of a graph $G=(V, E)$ of order $p$ and size $q$ is a bijection $f: V \cup E \rightarrow$ $\{i\}_{i=1}^{p+q}$ such that: (1) $f(u)+f(u v)+f(v)=k$ for all $u v \in E$; and (2) $f(V)=\{i\}_{i=1}^{p}$. Furthermore, when $G$ is a linear forest, the super edge-magic labeling of $G$ is called strong if it has the extra property that if $u v \in E(G), u^{\prime}, v^{\prime} \in V(G)$ and $d_{G}\left(u, u^{\prime}\right)=d_{G}\left(v, v^{\prime}\right)<+\infty$, then $f(u)+f(v)=f\left(u^{\prime}\right)+f\left(v^{\prime}\right)$. In this paper we introduce the concept of strong super edge-magic labeling of a graph $G$ with respect to a linear forest $F$, and we study the super edge-magicness of an odd union of nonnecessarily isomorphic acyclic graphs. Furthermore, we find exponential lower bounds for the number of super edge-magic labelings of these unions. The case when $G$ is not acyclic will be also considered.
\end{abstract}

2010 Mathematics subject classification: primary 05C78.

Keywords and phrases: super edge-magic labeling, strong super edge-magic labeling.

\section{Introduction}

Graphs considered in this paper are not necessarily simple, that is, they may contain loops but multiple edges are not allowed. Also, for most of the graph theory terminology and notation utilized, we refer the reader to either Chartrand and Lesniak [7] or to West [16]. However, in order to make the paper reasonably self contained we mention that by $G=(V, E)$ we mean a graph with vertex set $V$ and with edge set $E$. Also the notation $V(G)$ and $E(G)$ will be used in order to denote the vertex set and the edge set respectively of a given graph $G$. A $(p, q)$-graph is a graph of order $p$ and size $q$.

For either a graph or a digraph $D$, we will denote its adjacency matrix by $A(D)$ and for a digraph $D$ the notation und $(D)$ denotes the underlying graph of $D$. The seminal paper on edge-magic labelings was published in 1970 by Kotzig and Rosa [11] who called these labelings magic valuations. These were later rediscovered by Ringel and Lladó [14] who coined one of the most popular terms for them in current use: edge-magic labelings. More recently, they have also been referred to as edgemagic total labelings by Wallis [15]. For a $(p, q)$-graph $G$, a bijective function

The research conducted in this document by second and forth author has been supported by the Spanish Research Council under project MTM2008-06620-C03-01 and by the Catalan Research Council under grant 2009SGR1387.

(C) 2011 Australian Mathematical Publishing Association Inc. 0004-9727/2011 \$16.00 
$f: V(G) \cup E(G) \rightarrow\{1,2, \ldots, p+q\}$ is an edge-magic labeling of $G$ if for each $u v \in E(G), f(u)+f(u v)+f(v)$ is a constant $k$ called the valence of $f$. If such a labeling exists, then $G$ is said to be an edge-magic graph. In [8], Enomoto et al. defined an edge-magic labeling $f$ of a graph $G$ to be a super edge-magic labeling of $G$ if $f$ has the additional property that $f(V(G))=\{i\}_{i=1}^{p}$. It is worthwhile mentioning that an equivalent labeling had already been introduced by Acharya and Hedge in [1] under the name of strongly indexable labeling. A super edge-magic graph is a graph that admits a super edge-magic labeling. Super edge-magic graphs have been called strong edge-magic total graphs by Wallis [15] although in this paper we will use the term strong to denote a different concept, in the same manner as Bača et al. in [4].

Let $G=(V, E)$ be a $(p, q)$-linear forest. Assume that

$$
f: V \cup E \rightarrow\{1,2, \ldots, p+q\}
$$

is a super edge-magic labeling of $G$ with the extra property that if $u v \in E, u^{\prime}, v^{\prime} \in$ $V(G)$ and $d_{G}\left(u, u^{\prime}\right)=d_{G}\left(v, v^{\prime}\right)<+\infty$, then we have that $f(u)+f(v)=f\left(u^{\prime}\right)+$ $f\left(v^{\prime}\right)$. In this case, the labeling $f$ is said to be a strong super edge-magic labeling of $G$ and $G$ a strong super edge-magic graph.

In this paper, following a similar reasoning, we define the concept of strong super edge-magic labeling of a graph with respect to a linear forest. Let $G=(V, E)$ be a $(p, q)$-graph and let $F$ be any linear forest contained in $G$. A strong super edge-magic labeling of $G$ with respect to $F$ is a super edge-magic labeling $f$ of $G$ with the extra property that if $u v \in E(F), u^{\prime}, v^{\prime} \in V(F)$ and $d_{F}\left(u, u^{\prime}\right)=d_{F}\left(v, v^{\prime}\right)<+\infty$ then we have that $f(u)+f(v)=f\left(u^{\prime}\right)+f\left(v^{\prime}\right)$. If a graph $G$ admits a strong super edgemagic labeling with respect to some linear forest $F$, then we say that $G$ is a strong super edge-magic graph with respect to $F$.

At this point, we define the complementary labeling $\bar{f}$ of a strong super edgemagic labeling with respect to a linear forest. Let $G=(V, E)$ be a $(p, q)$-graph and let $f: V \cup E \rightarrow\{1,2, \ldots, p+q\}$ be a strong super edge-magic labeling of $G$ with respect to some linear forest $F$. Then the complementary labeling of $f$, denoted by $\bar{f}$, is the labeling defined by the rule

$$
\bar{f}(x)= \begin{cases}p+1-f(x), & \forall x \in V(G), \\ 2 p+1+q-f(x), & \forall x \in E(G) .\end{cases}
$$

The following result is an easy observation.

REMARK 1.1. Let $G=(V, E)$ be a $(p, q)$-graph and let $f: V \cup E \rightarrow\{1,2, \ldots$, $p+q$ \} be a strong super edge-magic labeling of $G$ with respect to some linear forest $F$ in $G$. Then the complementary labeling $\bar{f}$ is also a strong super edge-magic labeling of $G$ with respect to $F$.

Next, we state the following result by Figueroa-Centeno et al. [9]

LEMMA 1.2 [9]. A $(p, q)$-graph $G$ is super edge-magic if and only if there exists a bijective function $f: V(G) \rightarrow\{1,2, \ldots, p\}$ such that the set $S=\{f(u)+f(v)$ : $u v \in E(G)\}$ consists of $q$ consecutive integers. In such a case, $f$ can be extended to 
a super edge-magic labeling of $G$ with valence $k=p+q+s$ where $s=\min (S)$ and $S=\{k-(p+1), k-(p+2), \ldots, k-(p+q)\}$.

At this point, we will state an adaptation of Lemma 1.2 for strong super edge-magic labelings with respect to a linear forest.

LEMMA 1.3. A $(p, q)$-graph $G$ is strong super edge-magic with respect to a linear forest $F$ if and only if there exists a bijective function $f: V(G) \rightarrow\{1,2, \ldots, p\}$ such that the following conditions apply.

(1) The set $S=\{f(u)+f(v): u v \in E(G)\}$ consists of $q$ consecutive integers.

(2) If $u v \in E(F), u^{\prime}, v^{\prime} \in V(F)$ and $d_{F}\left(u^{\prime}, u\right)=d_{F}\left(v^{\prime}, v\right)<+\infty$, then $f(u)+$ $f(v)=f\left(u^{\prime}\right)+f\left(v^{\prime}\right)$.

In such a case, $f$ can be extended to a strong super edge-magic labeling with respect to a linear forest $F$ in $G$ with valence $k=p+q+s$ where $s=\min (S)$ and $S=\{k-(p+1), k-(p+2), \ldots, k-(p+q)\}$.

PROOF. The lemma follows immediately from Lemma 1.2 and the definition of strong super edge-magic labeling with respect to a linear forest.

Hence, due to Lemma 1.3, it is sufficient to exhibit the vertex labels of a strong super edge-magic labeling of $G$ with respect to a linear forest $F$.

In his $\mathrm{PhD}$ thesis, Barrientos [6] introduced the concept of a path-like tree as follows.

We embed the path $P_{n}$ as a subgraph of the two-dimensional grid, this is to say, the cartesian product $P_{r} \times P_{t}$ of a path on $r$ vertices with a path of $t$ vertices. Given such an embedding we consider the ordered set of subpaths $L_{1}, L_{2}, \ldots, L_{h}$ which are maximum straight segments in the embedding, where the end of $L_{i}$ is the beginning of $L_{i+1}$ for any $i=1,2, \ldots, h-1$. Suppose that $L_{i} \cong P_{2}$ for some $i \in\{2, \ldots, h-1\}$, $V\left(L_{i}\right)=\left\{u_{0}, v_{0}\right\}$, thus $u_{0} \in V\left(L_{i-1}\right) \cap V\left(L_{i}\right)$ and $v_{0} \in V\left(L_{i}\right) \cap V\left(L_{i+1}\right)$. Let $u \in$ $V\left(L_{i-1}\right) \backslash\left\{u_{0}\right\}$ and $v \in V\left(L_{i+1}\right) \backslash\left\{v_{0}\right\}$, such that the distance of $u$ and $v$ in the grid is 1 . The replacement of the edge $u_{0} v_{0}$ by the new edge $u v$ is called an elementary transformation of the path $P_{n}$. We say that a tree $T$ of order $n$ is a path-like tree, when it can be obtained after a sequence of elementary transformations of an embedding of $P_{n}$ in the two-dimensional grid.

The labeling properties of path-like trees and the union of them have been studied by many authors lately, for instance see [2, 3, 5, 6, 12, 13].

Motivated by the concept of path-like trees, now we introduce the concept of a derived graph of a graph $G$ with respect to a linear forest $F$ and, in this paper, we consider paths to be also linear forests. Let $G$ be a graph and let $F$ be any linear forest in $G$. Consider the set $\mathfrak{S}$ of all unions of path-like trees that can be obtained by elementary transformations of $F$. That is to say, let $F=\bigcup_{k} P^{k}$, where $P^{k}$ is a path. Then $\bigcup_{k} T^{k} \in \mathfrak{S}$ if and only if $T^{k}$ is a path-like tree that can be obtained by elementary transformations of an embedding of $P^{k}$ in the two-dimensional grid. A derived graph of $G$ with respect to $F$ is a graph $G^{\prime}$ with $V\left(G^{\prime}\right)=V(G) \wedge E\left(G^{\prime}\right)=$ $(E(G) \backslash E(F)) \cup E(S)$, for some $S \in \mathfrak{S}$. The set of all derived graphs of $G$ with respect to $F$ is denoted by $d G / d F$. (See Figure 1 for illustration.) 

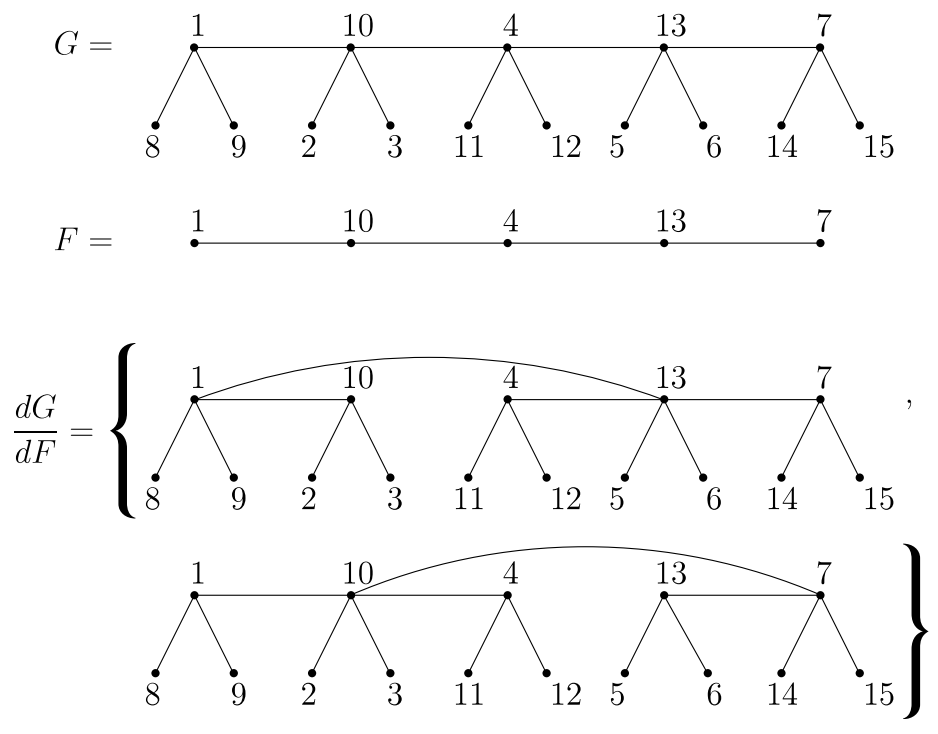

FIGURE 1. An illustration of the set of derived graphs.

The main goal in this paper is to prove the following statement.

THEOREM 1.4. Let $m$ be an odd integer. Let $G$ be an acyclic graph which is strong super edge-magic with respect to a linear forest $F$. Then $m G$ is strong super edgemagic with respect to $\bigcup_{i=1}^{m} F_{i}$, where $F_{i} \simeq F$ for $i=1,2, \ldots, m$.

Furthermore, $m G$ admits an exponential number of strong super edge-magic labelings with respect to the linear forest $F_{1} \cup F_{2} \cup \cdots \cup F_{m}$. As a corollary we also find that if $G$ is an acyclic strong super edge-magic graph with respect to a linear forest $F$, then an arbitrary odd union of graphs in the set $d G / d F$ is super edge-magic.

In order to achieve our goal, we use a modification of the technique introduced in [4] that has been also used in [12]. The key point in this technique is a digraph operation that was defined by Figueroa et al. in [10]. Let $D$ be a digraph and let $\Gamma=\left\{F_{1}, F_{2}, \ldots, F_{s}\right\}$ be a family of digraphs such that $V\left(F_{i}\right)=V$ for every $i=$ $1,2, \ldots, s$. Consider any function $h: E(D) \rightarrow \Gamma$. Then the product $D \otimes_{h} \Gamma$ is the digraph with vertex set $V\left(D \otimes_{h} \Gamma\right)=V(D) \times V$ and

$$
((a, b),(c, d)) \in E\left(D \otimes_{h} \Gamma\right) \Leftrightarrow[(a, c) \in E(D) \wedge(b, d) \in E(h(a, c))] .
$$

Notice that the adjacency matrix of $D \otimes_{h} \Gamma$, denoted by $A\left(D \otimes_{h} \Gamma\right)$, can be obtained by replacing every 0 entry of $A(D)$ by the $|V| \times|V|$ null square matrix and if there is a 1 in position $(a, c)$ then we replace this 1 by $A(h(a, c))$. Notice that when $h$ is a constant function, then we have the classical Kronecker product of two matrices. 
In [10] Figueroa-Centeno et al. called a digraph $D$ super edge-magic if und $(D)$ is super edge-magic. In general, when we say that a digraph $D$ admits any labeling $f$, we mean that its underlying graph admits the labeling $f$. In the same paper, the authors proved the following two results.

THEOREM 1.5 [10]. Let $D$ be a super edge-magic digraph. Let $\Gamma_{m}=\left\{R_{i}\right\}_{i=1}^{s}$ be the family of all super edge-magic 1-regular labeled digraphs (each $R_{i}$ is either a cycle or a union of cycles where each component has been oriented cyclically for $i=1,2, \ldots, s)$ of the same odd order $m$. Consider any function $h: E(D) \rightarrow \Gamma_{m}$. Then the digraph $D \otimes_{h} \Gamma_{m}$ is super edge-magic.

Assume that in $D$ and in $\Gamma_{m}$ each vertex takes the name of its label assigned by a super edge-magic labeling. The labeling they used to prove the super edge-magicness of $D \otimes_{h} \Gamma_{m}$ assigned the label $m(i-1)+j$ to the vertex $(i, j)$.

THEOREM 1.6 [10]. Let $\vec{T}$ be an oriented tree. Let $\Gamma_{m}=\left\{R_{i}\right\}_{i=1}^{s}$ be the family of 1-regular digraphs of order $m$. Consider any function $h: E(\vec{T}) \rightarrow \Gamma_{m}$. Then we have $\operatorname{und}\left(\vec{T} \otimes_{h} \Gamma_{m}\right)=m T$.

Also the following result found in [4] will be helpful in this paper.

THEOREM 1.7 [4]. Let $C_{n}$ be a cycle on $n$ vertices, $n \geq 11$ odd. The number of nonisomorphic super edge-magic labelings of $C_{n}$ is at least $\frac{5}{4} 2^{\lfloor n / 3\rfloor}+1$.

\section{Trees and forests}

In this section, we describe an algorithm that allows us to create odd unions of isomorphic trees or forests, which are strong super edge-magic with respect to some linear forest. We introduce the algorithm next.

Input. Let $G$ be an acyclic graph and let $f$ be a strong super edge-magic labeling of $G$ with respect to some linear forest $F$. Assume that each vertex takes the name of the label assigned by $f$.

(1) Orient the acyclic graph $\vec{G}$ in such a way that each path in $F$ is oriented from one leaf to the other.

(2) Let $\Gamma_{m}=\left\{R_{1}, R_{1}^{\prime}, R_{2}, R_{2}^{\prime}, \ldots, R_{s / 2}, R_{s / 2}^{\prime}\right\}$ be the family of all 1-regular digraphs of order $m=2 k+1$ for some positive integer $k$ labeled in a super edge-magic way. Assume that each vertex takes the name of its label. Each couple $\left(R_{j}, R_{j}^{\prime}\right), j=1, \ldots, s / 2$ comes from the same super edge-magic labeled digraph but with opposite orientation. That is to say, if a component is orientated clockwise in $R_{j}$, then the corresponding component in $R_{j}^{\prime}$ is oriented counter clockwise and vice versa.

(3) Consider a function $h: E(\vec{G}) \rightarrow \Gamma_{m}$ with $h(E(\vec{P})) \in\left\{R_{j}, R_{j}^{\prime}\right\}$ in every path $P$ of $F$, such that two consecutive arcs in $F$, namely $(x, y),(y, z)$, have $h(x, y) \neq h(y, z)$. 


\section{Algorithm 1.}

(1) Compute $\vec{G} \otimes_{h} \Gamma_{m}=\vec{Q}$.

(2) Take und $(\vec{Q})=Q$.

\section{Output.}

(1) The output is $Q=m G$ labeled in a strong super edge-magic way with respect to the linear forest $\bigcup_{i=1}^{m} F_{i}$ where $F_{i}$ is the corresponding copy of $F$ in each of the $m$ copies of $G, i=1,2, \ldots, m$.

PROOF OF THEOREM 1.4. It was proved in [10] that this algorithm gives a super edge-magic labeling $g$ of $m G$. The labeling can be obtained by assigning the label $m(i-1)+j$ to the vertex $(i, j)$ of the product. Thus, what remains to prove is the strong property of $g$ with respect to the linear forest $\bigcup_{i=1}^{m} F_{i}$. Let $\bar{F}=\bigcup_{i=1}^{m} F_{i}$.

Let $((u, q),(v, r)) \in E(\bar{F}),\left(u^{\prime}, s\right),\left(v^{\prime}, t\right) \in V(\bar{F})$ and assume that

$$
d_{\bar{F}}\left((u, q),\left(u^{\prime}, s\right)\right)=d_{\bar{F}}\left((v, r),\left(v^{\prime}, t\right)\right)<+\infty .
$$

Since every two consecutive arcs in $F$ have different image in $\left\{\overrightarrow{R_{j}}, \overleftarrow{R_{j}}\right\}$ we distinguish two cases.

Case 1. If $d_{\bar{F}}\left((u, q),\left(u^{\prime}, s\right)\right)=d_{\bar{F}}\left((v, r),\left(v^{\prime}, t\right)\right)$ is odd then, $s=r$ and $t=q$. Hence, we obtain that

$$
\begin{aligned}
g\left(\left(u^{\prime}, r\right)\right)+g\left(\left(v^{\prime}, q\right)\right) & =m\left(u^{\prime}+v^{\prime}-2\right)+r+q \\
& =m(u+v-2)+r+q=g((u, q))+g((v, r)) .
\end{aligned}
$$

Case 2. If $d_{\bar{F}}\left((u, q),\left(u^{\prime}, s\right)\right)=d_{\bar{F}}\left((v, r),\left(v^{\prime}, t\right)\right)$ is even then $s=q$ and $t=r$ and we proceed as in Case 1.

Next, we will see how this algorithm applies nicely to the following type of caterpillar.

Let $T$ be a caterpillar. The spine of $T$, denoted by $\operatorname{sp}(T)$, is the path that remains after eliminating all pendent edges of $T$. We define a caterpillar $T$ to be regular if each vertex of $\operatorname{sp}(T)$ is adjacent to the same number of pendent edges. Then we have the following proposition.

PROPOSITION 2.1. Every regular caterpillar $T$ is strong super edge-magic with respect to its spine $\mathrm{sp}(T)$.

PROOF. Let $T$ be the regular caterpillar with vertex set,

$$
V(T)=\left\{u_{i}: 1 \leq i \leq k\right\} \cup\left\{v_{i}^{j}: 1 \leq i \leq k, 1 \leq j \leq n\right\}
$$

and edge set

$$
E(T)=\left\{u_{i} u_{i+1}: 1 \leq i \leq k-1\right\} \cup\left\{u_{i} v_{i}^{j}: 1 \leq i \leq k, 1 \leq j \leq n\right\} .
$$




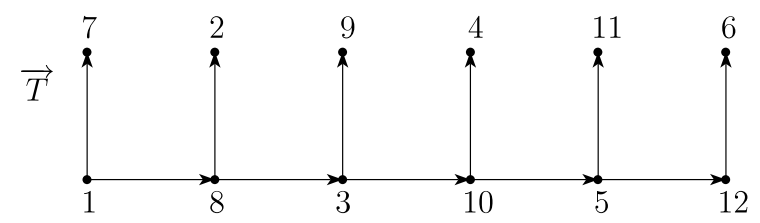

FIGURE 2. A strong super edge-magic labeling of a regular caterpillar $\vec{T}$.

Each vertex of $\operatorname{sp}(T)$ is adjacent to $n$ leaves. Let $f: V(T) \rightarrow\{1,2, \ldots, k+k n\}$ be the labeling defined as follows:

$$
f(v)= \begin{cases}1+(l-1)(n+1) & \text { if } v=u_{2 l-1}, \\ 1+j+(l-1)(n+1) & \text { if } v=v_{2 l}^{j}, \\ 1+j+\left\lfloor\frac{k-1}{2}\right\rfloor(n+1)+\frac{1+(-1)^{k}}{2} n+(l-1)(n+1) & \text { if } v=v_{2 l-1}^{j}, \\ 2+n+\left\lfloor\frac{k-1}{2}\right\rfloor(n+1)+\frac{1+(-1)^{k}}{2} n+(l-1)(n+1) & \text { if } v=u_{2 l},\end{cases}
$$

where $1 \leq 2 l-1,2 l \leq k$.

This labeling was used by Kotzig and Rosa in [11] to prove the edge-magicness of caterpillars. Moreover, $f$ is a strong super edge-magic labeling of $T_{n, l}^{k}$ with respect to its spine, since every two alternating vertices on the spine form an arithmetic progression.

From the previous result we obtain the following corollary.

COROLlARY 2.2. Let $T$ be any regular caterpillar. Then every tree $T^{\prime} \in d T / d[\operatorname{sp}(T)]$ is a super edge-magic graph.

PROOF. Since $T$ is strong super edge-magic with respect to its spine, every elementary transformation of an embedding of the spine in the two-dimensional grid gives a super edge-magic graph.

In particular, from the Proposition 2.1 and the algorithm, we obtain the following corollary.

COROLLARY 2.3. Let $T$ be a regular caterpillar. Then any odd union of graphs in the set $d T / d[\operatorname{sp}(T)]$ is a super edge-magic graph.

The following example illustrates the construction.

EXAMPLE 2.4. Let $\vec{T}$ be the oriented regular caterpillar shown in Figure 2. Note that each vertex takes the labels of the strong super edge-magic labeling with respect to its spine described in Proposition 2.1.

Let $\Gamma_{3}=\{A, B\}$ be the family of all 1-regular digraphs of order three (Figure 3). 

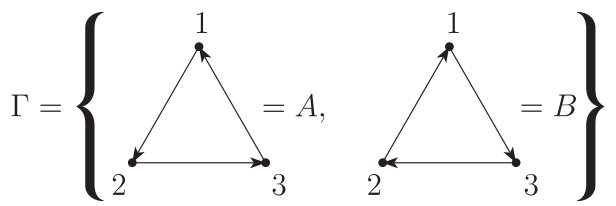

FIGURE 3. The family of all 1-regular digraphs of order 3.
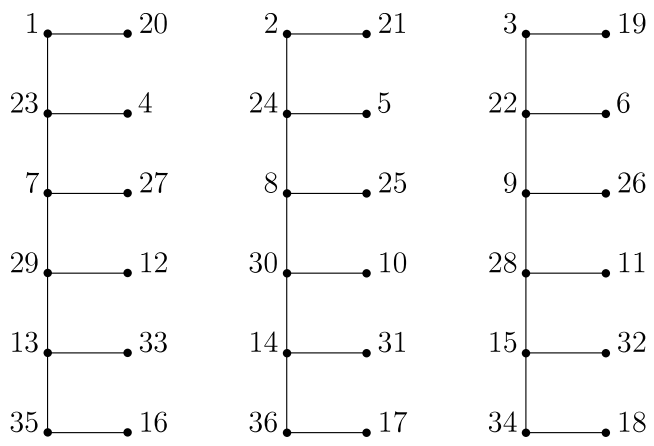

FIGURE 4 . The graph obtained by replacing the arcs by edges in $\vec{T} \otimes_{h} \Gamma_{3}$.

Assume that $h: E(T) \longrightarrow \Gamma_{3}$ is the function defined by

$$
\left\{\begin{array}{llll}
h(1,8)=A, & h(8,3)=B, & h(3,10)=A, & h(10,5)=B, \\
h(5,12)=A, & h(1,7)=A, & h(8,2)=B, & h(3,9)=B, \\
h(10,4)=A, & h(5,11)=B, & h(12,6)=B . &
\end{array}\right.
$$

Then, the graph und $\left.\vec{T} \otimes_{h} \Gamma_{3}\right)=3 T$ is strong super edge-magic with respect to $\bigcup_{i=1}^{3} F_{i}$, where $F_{i} \simeq \operatorname{sp}(T)$ for $i=1,2,3$. A super edge-magic labeling of und $\left(\vec{T} \otimes_{h} \Gamma_{3}\right)$ can be obtained by assigning the label $3(i-1)+j$ to the vertex $(i, j)$. (Figure 4.)

Moreover, if $T_{1}, T_{2}, T_{3} \in d T / d[\operatorname{sp}(T)]$ then the graph $G \cong T_{1} \cup T_{2} \cup T_{3}$ is super edge-magic. An example is shown in Figure 5.

Notice that when we are applying the algorithm, there are many functions $h$ that we can use, so that each one of them will produce a strong super edge-magic labeling of the resulting graph. In [10] it was shown that all the labelings that we obtain in this manner are not isomorphic. Now, let us find a lower bound for the number of functions $h$ that we can use.

Let $D$ be an acyclic digraph that is strong super edge-magic with respect to some linear forest $F$. Let $|E(D)|=q$ and $|E(F)|=q_{F}$. We denote by $c(F)$ the number of connected components of $F$. Assume that we are applying Algorithm 1 with the set $\Gamma_{m}$ of all 1-regular super edge-magic labeled digraphs of odd order $m$. Now, if $m \geq 11$, we know that the number of super edge-magic labelings of $C_{m}$ is lower 

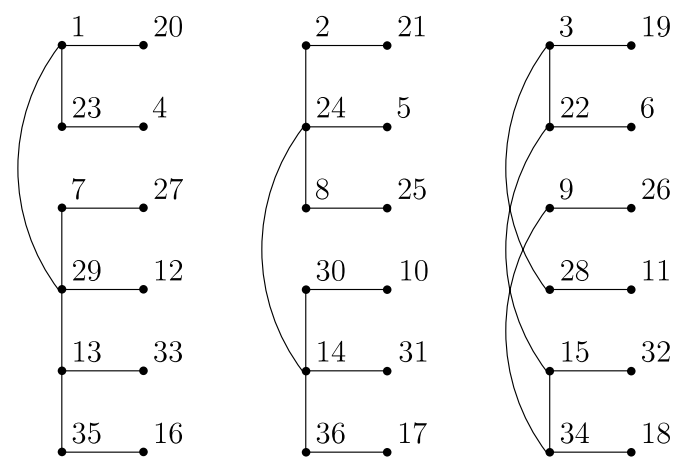

FIGURE 5. A super edge-magic labeled graph that can be obtained from $3 T$.

bounded by $\frac{5}{4} \cdot 2^{\lfloor m / 3\rfloor}+1$ by Theorem 1.7 . Hence $\left|\Gamma_{m}\right| \geq \frac{5}{2} \cdot 2^{\lfloor m / 3\rfloor}+2$ since each cycle $C_{m}$ admits two strong orientations. Now, every arc in $E(D)$ which is not an arc of $F$, can be assigned any of these $\frac{5}{2} \cdot 2^{\lfloor m / 3\rfloor}+2$ digraphs. When we choose a digraph for an arc of a path of the linear forest, then the rest of the images of the arcs in the path depend on this choice. So we have at least $\left(\frac{5}{2} \cdot 2^{\lfloor m / 3\rfloor}+2\right)^{c(F)}$ ways of choosing the images of the arcs of $F$. Thus, in total we have at least

$$
\left(\frac{5}{2} \cdot 2^{\lfloor m / 3\rfloor}+2\right)^{q-q_{F}+c(F)}
$$

distinct functions, and hence we have at least

$$
\left(\frac{5}{2} \cdot 2^{\lfloor m / 3\rfloor}+2\right)^{q-q_{F}+c(F)}
$$

different strong super edge-magic labelings of the resulting graph. Therefore we obtain the next theorem.

THEOREM 2.5. Let $G$ be an acyclic graph which is strong super edge-magic with respect to a linear forest $F$. Let $|E(G)|=q,|E(F)|=q_{F}$ and let $c(F)$ be the number of connected components of $F$. Then, any odd union of graphs in the set $d T / d[\operatorname{sp}(T)]$ admits at least

$$
\left(\frac{5}{2} \cdot 2^{\lfloor m / 3\rfloor}+2\right)^{q-q_{F}+c(F)}
$$

nonisomorphic super edge-magic labelings, where $m$ is the number of the graphs in the union.

Next, we consider another family of trees that we will denote by $T_{n, l}^{k}$.

Proposition 2.6. Let $T_{n, l}^{k}$ be the graph obtained from a path $F=u_{1} u_{2} \cdots u_{k}$ of $k$-vertices and $k$ paths $\left\{P^{i}\right\}_{i=1, \ldots, k}$ of $n$-vertices by identifying a vertex of $P^{i}$ at distance $l-1$ from a leaf with the vertex $u_{i}$ for $i=1, \ldots, k$. Then the graph $T_{n, l}^{k}$ is strong super edge-magic with respect to $F$ and with respect to $\bigcup_{i=1}^{k} P^{i}$. 


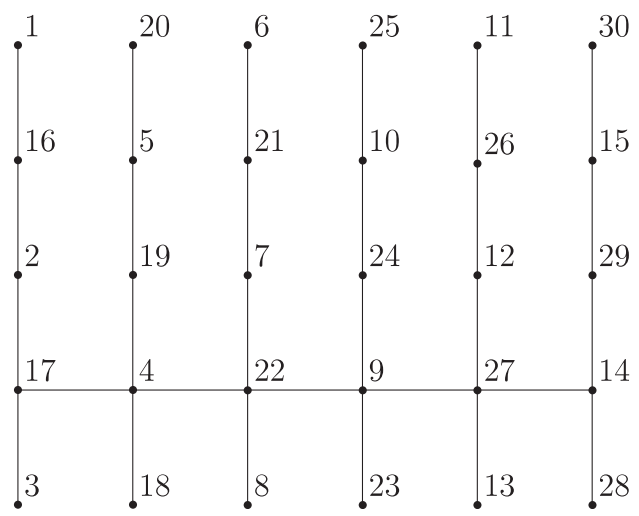

FIGURE 6. The graph $T_{5,4}^{6}$.

ProOf. Let $P^{i}=v_{1}^{i} v_{2}^{i} \cdots v_{n}^{i}$ for each $i=1, \ldots, k$. Let $P$ be the path obtained from $\bigcup_{i=1}^{k} P^{i}$ by adding the edges $\left\{v_{n}^{i} v_{1}^{i+1}: i=1, \ldots, k-1\right\}$. Let $f: V(P) \rightarrow$ $\{1, \ldots, n k\}$ be the labeling defined by

$$
f\left(v_{j}^{i}\right)= \begin{cases}\frac{n(i-1)+j+1}{2} & \text { for } n(i-1)+j \text { odd, } \\ \left\lceil\frac{n k}{2}\right\rceil+\frac{n(i-1)+j}{2} & \text { for } n(i-1)+j \text { even. }\end{cases}
$$

Notice that this labeling is one of the two possible strong labelings of a path [12]. Also we can embed the path $P$ into the two-dimensional grid $P_{k} \times P_{n}$. From this embedding we can recover the tree $T_{n, l}^{k}$ by elementary transformations. In particular, $F$ is the path $v_{l}^{1} v_{n+1-l}^{2} v_{l}^{3} v_{n+1-l}^{4} \cdots$. By construction, $f$ is also a super edge-magic labeling of $T_{n, l}^{k}$. What remains to prove is that this labeling is strong with either $F$ or $\bigcup_{i} P^{i}$. However, this is clear since for each path the labels of alternating vertices form an arithmetic progression, of difference 1 in $P^{i}$ and of difference $n$ in $F$.

Figure 6 shows the graph $T_{5,4}^{6}$ with the labeling described in the proof of Proposition 2.6.

COROLlary 2.7. With the same notation as before. For all positive integers $l, m, n$ and $k$, the graph $(2 m+1) T_{n, l}^{k}$ is super edge-magic with respect to the linear forest $(2 m+1) F$ and with respect to $\bigcup_{i=1}^{k}(2 m+1) P^{i}$.

PROOF. Since $T_{n, l}^{k}$ is an acyclic strong super edge-magic graph with respect to $F$, by Theorem 1.4 we obtain the result.

\section{Graphs with cycles}

The goal of this section is to generalize the algorithm introduced in the previous section to graphs with cycles. We begin this section by introducing an example. 


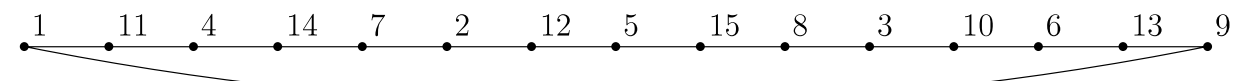

FIGURE 7. A strong super edge-magic labeling with respect $F=G^{\prime} \backslash\{\{2,7\},\{3,8\},\{1,9\}\}$.

EXAmple 3.1. Let $G$ be the oriented cycle $1 \rightarrow 4 \rightarrow 2 \rightarrow 5 \rightarrow 3 \rightarrow 1$ and let $\Gamma_{3}=$ $\{A, B\}$ be the family of all 1-regular digraphs of order three (see Figure 3). Note that, $G$ is strong super edge-magic with respect to the path $F$ defined as $V(F)=V(G)$, $E(F)=E(G) \backslash\{(3,1)\}$.

Let $h: E(G) \longrightarrow\{A, B\}$ be the function defined by

$$
h(1,4)=A, \quad h(4,2)=B, \quad h(2,5)=A, \quad h(5,3)=B, \quad h(3,1)=A .
$$

Then the graph $G^{\prime}=\operatorname{und}\left(G \otimes_{h} \Gamma_{3}\right)$ is strong super edge-magic with respect to $\bar{F}$, where $\bar{F}$ is the linear forest obtained from $G \otimes_{h} \Gamma_{3}$ by removing the arcs

$$
\{((3,1),(1,2)),((3,2),(1,3)),((3,3),(1,1))\} .
$$

Notice that, $\bar{F}=F \otimes_{h} \Gamma_{3}$. A strong super edge-magic labeling of $G^{\prime}$ with respect to $\bar{F}$ can be obtained by assigning the label $3(i-1)+j$ to the vertex $(i, j)$ of the product (see Figure 7). Moreover, any sequence of elementary transformations in the paths of $\bar{F}$ gives a super edge-magic graph.

Our goal is to generalize this example to any graph which contains cycles as subgraphs and that is strong super edge-magic with respect to some linear forest.

THEOREM 3.2. Let $G$ be a graph that is strong super edge-magic with respect to some linear forest $F$. Let $\Gamma_{m}$ be the family of all 1-regular digraphs of odd order $m$. Consider any orientation of $G$, namely $\vec{G}$, such that in each component of $F$ it is possible to travel from one leaf to the other following the direction of the arrows. Consider a function $h: E(\vec{G}) \rightarrow \Gamma_{m}$ with $h(E(\vec{F})) \in\left\{R_{i}, R_{i}^{\prime}\right\}$ such that two consecutive arcs in $\vec{F}$ namely $(x, y),(y, z)$ have $h(x, y) \neq h(y, z)$. Then the graph und $\left(\vec{G} \otimes_{h} \Gamma_{m}\right)$ is strong super edge-magic with respect to $\bigcup_{i=1}^{m} F_{i}$, where $F_{i} \simeq F$ for $i=1, \ldots, m$.

PROOF. We know that $\vec{G} \otimes_{h} \Gamma_{m}$ is super edge-magic by Theorem 1.5 and that, since $F$ is acyclic, by Theorem 1.4 we obtain that und $\left(\vec{F} \otimes_{h_{\mid E(F)}} \Gamma_{m}\right)=\bigcup_{i=1}^{m} F_{i}$, where $F_{i} \simeq F$ for $i=1, \ldots, m$. Moreover, assuming that each vertex takes the name of its label by a super edge-magic labeling, we know that the labeling that assigns the label $m(i-1)+j$ to the vertex $(i, j)$ of the product produces a super edge-magic labeling of $\vec{G} \otimes_{h} \Gamma_{m}$. Finally, by the same argument as in the proof of Theorem 1.4, we obtain the strong property of $f$ with respect to the linear forest $\bigcup_{i=1}^{m} F_{i}$. 


\section{References}

[1] B. D. Acharya and S. M. Hegde, 'Strongly indexable graphs', Discrete Math. 93 (1991), 123-129.

[2] M. Bača, Y. Lin and F. A. Muntaner-Batle, 'Super edge-antimagic labelings of the path-like trees', Util. Math. 73 (2007), 117-128.

[3] M. Bača, Y. Lin and F. A. Muntaner-Batle, 'Normalized embeddings of path-like trees', Util. Math. 78 (2009), 11-31.

[4] M. Bača, Y. Lin, F. A. Muntaner-Batle and M. Rius-Font, 'Strong labelings of linear forests', Acta Math. Sin. (Engl. Ser.) 25(12) (2009), 1951-1964.

[5] M. Bača and M. Miller, Super Edge-Antimagic Graphs (Brown Walker Press, Boca Raton, FL, 2008).

[6] C. Barrientos, 'Difference vertex labelings', PhD Thesis, Universitat Politècnica de Catalunya, 2004.

[7] G. Chartrand and L. Lesniak, Graphs and Digraphs, 3rd edn (CRC Press, Boca Raton, FL, 1996).

[8] H. Enomoto, A. S. Lladó, T. Nakamigawa and G. Ringel, 'Super edge-magic graphs', SUT J. Math. 34 (1998), 105-109.

[9] R. M. Figueroa-Centeno, R. Ichishima and F. A. Muntaner-Batle, 'The place of super edge-magic labeling among other classes of labeling', Discrete Math. 231 (2001), 153-168.

[10] R. M. Figueroa-Centeno, R. Ichishima, F. A. Muntaner-Batle and M. Rius-Font, 'Labeling generating matrices', J. Combin. Math. Combin. Comput. 67 (2008), 189-216.

[11] A. Kotzig and A. Rosa, 'Magic valuations of finite graphs', Canad. Math. Bull. 13 (1970), 451-461.

[12] S. C. López, F. A. Muntaner-Batle and M. Rius-Font, 'Enumerating super edge-magic labelings for some types of path-like trees', submitted.

[13] F. A. Muntaner-Batle and M. Rius-Font, 'On the structure of path-like trees', Discuss. Math. Graph Theory 28(2) (2008), 249-265.

[14] G. Ringel and A. Lladó, 'Another tree conjecture', Bull. Inst. Combin. Appl. 18 (1996), 83-85.

[15] W. D. Wallis, Magic Graphs (Birkhäuser, Boston, MA, 2001).

[16] D. B. West, Introduction to Graph Theory (Prentice Hall, Upper Saddle River, NJ, 1996).

A. AHMAD, Department of Mathematics, Govt. College University,

Lahore, Pakistan

e-mail: ahmadsms@gmail.com

S. C. LÓPEZ, Departament de Matemàtica Aplicada IV,

Universitat Politècnica de Catalunya, C/Esteve Terrades 5,

08860 Castelldefels, Spain

e-mail: susana@ma4.upc.edu

F. A. MUNTANER-BATLE, Graph Theory and Applications Research Group,

School of Electrical Engineering and Computer Science,

Faculty of Engineering and Built Environment,

The University of Newcastle, NSW 2308, Australia

e-mail: famb1es@yahoo.es

M. RIUS-FONT, Departament de Matemàtica Aplicada IV,

Universitat Politècnica de Catalunya, C/Esteve Terrades 5,

08860 Castelldefels, Spain

e-mail: mrius@ma4.upc.edu 